\title{
First Case of Nosocomial Fungemia Caused by Candida melibiosica
}

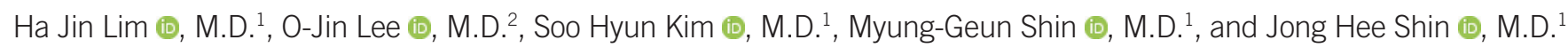

${ }^{1}$ Department of Laboratory Medicine, Chonnam National University Medical School, Gwangju, Korea; ${ }^{2}$ Department of Laboratory Medicine, Green Cross Laboratories, Yongin, Korea

\section{Dear Editor,}

Candida melibiosica is an unusual Candida species often isolated from rivers, oceans, and some traditional beers [1-3]. Human infection caused by this organism has never been described. We report a case of nosocomial fungemia by $C$. melibiosica in an intensive care unit (ICU) patient. To our knowledge, this is the first report to demonstrate that $C$. melibiosica causes nosocomial fungemia and can act as a human pathogen. This study was approved by the Institutional Review Board of Chonnam National University Hospital, Gwangju, Korea (IRB CNUH-2014290). A waiver of consent was granted as the study is retrospective in nature.

In December 2010, an 82-year-old man was admitted to emergency room of Chonnam National University Hospital owing to altered mental status. He suffered from aspiration pneumonia and hypoxic brain damage due to asphyxia. He had no known underlying disease. The patient was diagnosed as having nosocomial fungemia because the same yeast was repeatedly isolated from blood cultures collected on days 12, 15, 17, 18, and 22 of his 39 days of hospital stay. Once transferred to the
ICU on day 2, he was treated with tazobactam/piperacillin for 11 days, intravenous nutrition via central venous catheter (CVC) for 17 days, and mechanical ventilation for 38 days. On day 12 , he developed an intermittent fever (temperature, $37.8-38.8^{\circ} \mathrm{C}$ ) that persisted for 13 days. His leukocyte count and serum $\mathrm{C}$-reactive protein level on day 12 were $14.5 \times 10^{9} / \mathrm{L}$ (neutrophil count $12.9 \times 10^{9} / \mathrm{L}$ ) and $1,296.2 \mathrm{nmol} / \mathrm{L}$, respectively. The blood cultures were performed using about $10 \mathrm{~mL}$ of peripheral blood (PB) or catheter-drawn blood (CB). Nine of the 10 blood culture sets obtained from days 12 (one $\mathrm{PB}$ and one $\mathrm{CB}$ ), 15 (two PB), 17 (one PB and one $\mathrm{CB}$ ), 18 (two PB), and 22 (one of two $\mathrm{PBs}$ ) yielded yeast isolates with the same microscopic and colony morphological findings (Table 1). Fluconazole therapy (100 $\mathrm{mg} /$ day) was started on day 15 , and the CVC was removed on day 18. A culture of the removed CVC tip was negative, and the blood culture became negative on day 27 . The patient's condition improved, and he was transferred to another hospital.

Two isolates obtained on days 12 and 17 of the patient's hospital stay were analyzed by sequencing of the large-subunit rRNA gene (D1-D2 region); BLAST search results indicated a
Received: June 24, 2019

Revision received: July 12, 2019

Accepted: September 23, 2019

Corresponding author: Jong Hee Shin, M.D.

Department of Laboratory Medicine, Chonnam National University Medical School, 42 Jebong-ro, Dong-gu, Gwangju 61469, Korea

Tel: +82-62-220-5342, Fax: +82-62-224-2518

E-mail: shinjh@chonnam.ac.kr

Co-corresponding author: Soo Hyun Kim, M.D.

Department of Laboratory Medicine, Chonnam National University Medical School, 42 Jebong-ro, Dong-gu, Gwangju 61469, Korea

Tel: +82-61-379-7952, Fax: +82-61-379-7984

E-mail: alpinboy@chonnam.ac.kr 
Table 1. Morphological findings and identification results for blood isolates using biochemical methods, MALDI-TOF MS, and DNA sequence analysis

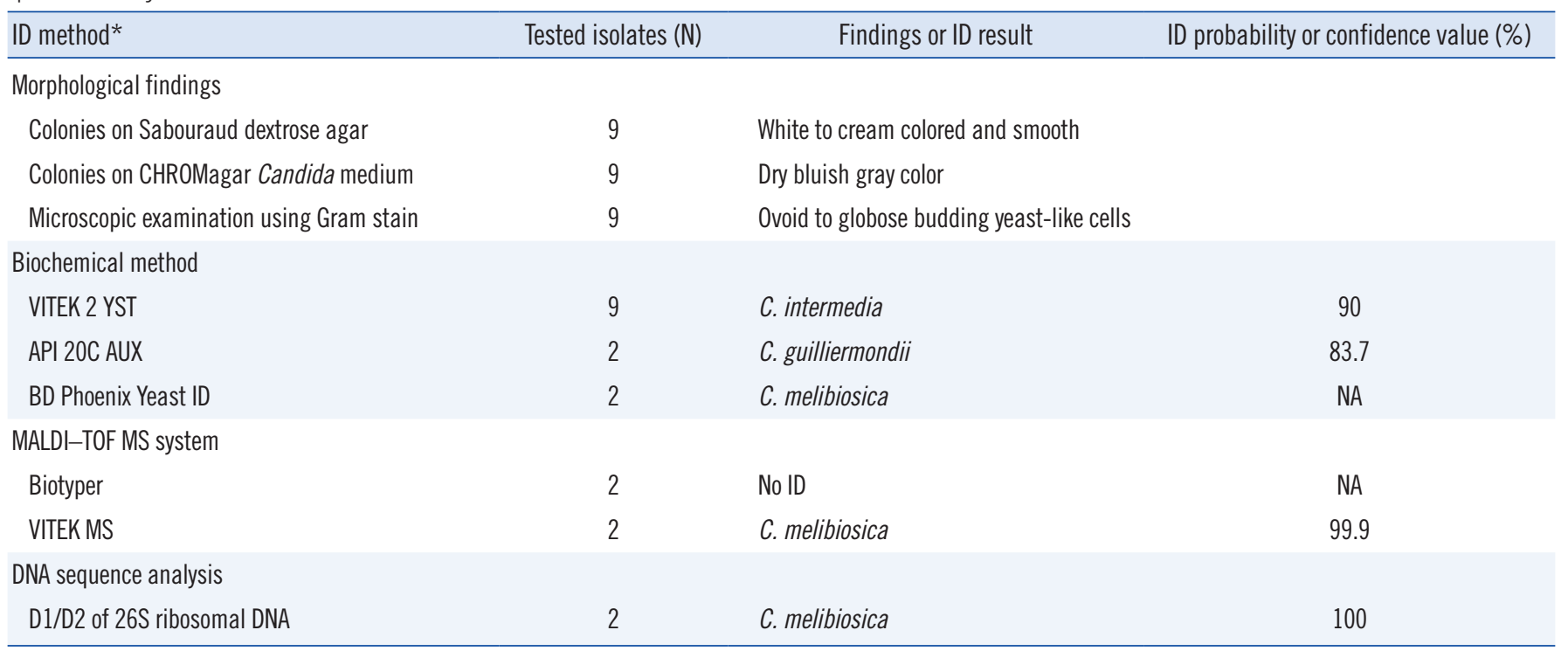

*Manufacturer details of respective methods are CHROMagar Candida medium (BBL, Becton Dickinson, Sparks, MD, USA), VITEK 2 YST (bioMérieux, Marcy l'Etoile, France), API 20C AUX (bioMérieux), BD Phoenix Yeast ID (BD Diagnostics, Sparks, MD, USA), Biotyper (Bruker Daltonics, Billerica, MA, USA), and VITEK MS (bioMérieux).

Abbreviations: MALDI-TOF MS, matrix-assisted laser desorption/ionization-time of flight mass spectrometry; ID, identification; NA, not available.

100\% match (499/499 bps) with C. melibiosica (GenBank accession no: U44813.1; Table 1). However, all nine (seven from PB and two from $\mathrm{CB}$ ) isolates were misidentified as $C$. intermedia by VITEK 2 YST (bioMérieux, Marcy l'Etoile, France), which was routinely used for Candida identification in our laboratory. Two blood isolates obtained on days 12 and 17 were correctly identified as $C$. melibiosica by the BD Phoenix Yeast ID panel (BD Diagnostics, Sparks, MD, USA), but they were misidentified as $C$. guilliermondii by API 20C AUX (bioMérieux). Of the two commercial matrix-assisted laser desorption/ionization-time of flight mass spectrometry (MALDI-TOF MS) systems, the VITEK MS (bioMérieux) with Knowledge Base version 3.0 correctly identified two isolates as C. melibiosica, while the MALDI-TOF Biotyper (Bruker Daltonics, Billerica, MA, USA) gave no results (no identification) because $C$. melibiosica was not included in the Biotyper database. The minimum inhibitory concentration (MIC) for fluconazole was $0.5 \mu \mathrm{g} / \mathrm{mL}$ using the Clinical and Laboratory Standards Institute (CLSI) M27-A3 broth microdilution method [4].

Infections by many uncommon Candida species have recently been reported [5]. This may be partly due to improvements in fungal identification in clinical microbiology, as well as an increase in patients at a higher risk of candidemia. Our case shows that $C$. melibiosica can be correctly identified by some commercially available systems, including the BD Phoenix and VITEK
MS systems, as well as through gene sequencing; however, some commercial systems misidentify it. A recent literature review revealed that, although rare, this organism is among the list of fungal species isolated from cultures of clinical specimens, including sputum [6] and blood [7, 8]. However, the full extent of this organism's potential for human infection is not completely known.

Candida species are part of the human microbiota in mucosa and skin, and most cases of fungemia involve endogenous flora [9]. However, because C. melibiosica is not commonly found in healthy individuals, it most likely entered through our patient's skin or mucosa. Although our patient had a CVC when the first positive cultures were obtained, it was difficult to trace the portal of entry of the organism because the CVC tip culture was negative. However, our case shows that ICU stay, long-term CVC use, broad-spectrum antibiotic use, and aging may increase the risk of infection with uncommon Candida species. The C. melibiosica isolates in our case showed low MICs for fluconazole $(0.5 \mu \mathrm{g} / \mathrm{mL})$, although the CLSI has not established species-specific clinical breakpoints. Considering that Candida encompasses a high diversity of species, each with its own behavior and predisposing factors for human infections [10], this report highlights the importance of reporting the identification and clinical features of rare Candida infections. 


\section{Author Contributions}

JHS and SHK conceived and designed the study; OJL and HJL collected and contributed to data; JHS, SHK, and HJL contributed to writing the final manuscript; MGS participated in coordination and discussion. All authors have accepted their responsibility for the entire content of this manuscript and approved submission.

\section{Conflicts of Interest}

No potential conflicts of interest relevant to this article were reported.

\section{Research Funding}

This research was supported by the Basic Science Research Program through the National Research Foundation of Korea (NRF) funded by the Ministry of Education (NRF-2019R1A2C1004644).

\section{ORCID}

Ha Jin Lim https://orcid.org/0000-0003-2436-5007

O-Jin Lee https://orcid.org/0000-0002-4003-071X Soo Hyun Kim https://orcid.org/0000-0001-9739-711X Myung-Geun Shin https://orcid.org/0000-0002-0372-9185 Jong Hee Shin

\section{REFERENCES}

1. Loureiro STA, Cavalcanti MADQ, Neves RP, Passavante JZDO. Yeasts isolated from sand and sea water in beaches of Olinda, Pernambuco state, Brazil. Braz J Microbiol 2005;36:333-7.

2. Maoura N, Mbaiguinam M, Nguyen HV, Gaillardin C, Pourquie J. Identification and typing of the yeast strains isolated from bili bili, a traditional sorghum beer of Chad. Afr J Biotechnol 2005;4:646-56.

3. Medeiros AO, Missagia BS, Brandão LR, Callisto M, Barbosa FA, Rosa CA. Water quality and diversity of yeasts from tropical lakes and rivers from the Rio Doce basin in Southeastern Brazil. Braz J Microbiol 2012; 43:1582-94.

4. CLSI. Reference method for broth dilution antifungal susceptibility testing of yeasts. 4th ed. CLSI standard M27. Wayne, PA: Clinical and Laboratory Standards Institute; 2017.

5. Miceli MH, Diaz JA, Lee SA. Emerging opportunistic yeast infections. Lancet Infect Dis 2011;11(2):142-51.

6. Buckley HR and van Uden N. Five new Candida species. Mycopathol Mycol Appl 1968;36:257-66.

7. Hamid SUB, Tan S, Ridzuan SNBA, Seman MSBC, Ramli R, Khaithir TMN. Antifungal susceptibility patterns among Candida species isolated from blood at Universiti Kebangsaan Malaysia Medical Centre. Sains Malays 2012;41:961-7.

8. Kim DK, Hwang GY, Yoo G, Kim J, Uh Y. Trend of prevalence and antifungal drug resistance of Candida species isolated from candidemia patients at a tertiary care hospital during recent two decades. Ann Clin Microbiol 2017;20:53-62.

9. Nucci M and Anaissie E. Revisiting the source of candidemia: skin or gut? Clin Infect Dis 2001;33:1959-67.

10. Bretagne S, Renaudat C, Desnos-Ollivier M, Sitbon K, Lortholary O, Dromer $F$, et al. Predisposing factors and outcome of uncommon yeast species-related fungaemia based on an exhaustive surveillance programme (2002-14). J Antimicrob Chemother 2017;72:1784-93. 